\title{
INNOVATIONEN
}

\section{Fördermittel einwerben für neue Konzepte}

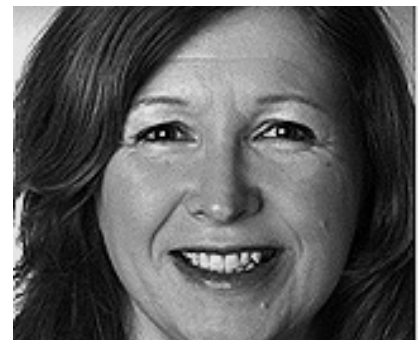

VON FRAUKE HENNINGS Frauke Hennings ist Organisationsberaterin bei dem Beratungsunternehmen contec $\mathrm{GmbH}$. Ihr fachlicher Schwerpunkt liegt in der strategischen Neuausrichtung, im Krisenmanagement und in der Personalentwicklung von Unternehmen der Behindertenhilfe.

Internet http://www.contec.de

\author{
Wer Innovationen durchsetzen will, braucht oft neues \\ Personal und frisches Geld. Mit einem stimmigen \\ "Businessplan " können Menschen und Finanziers \\ überzeugt werden, wie das Beispiel der Behinderten- \\ hilfe zeigt.
}

Die Zeichen stehen auf Sturm: Stationäre Wohnangebote der Behindertenhilfe werden in den meisten Bundesländern nicht mehr bewilligt, Kostenträger fordern Einrichtungen zur Konversion und zum Aufbau dezentraler ambulantisierter Angebote auf. Gesellschaftlich und politisch ist das Thema Inklusion mittlerweile in aller Munde und die Kostenträger stärken die personengebundene Förderung bereits heute durch individualisierte Gesamtplanund Bewilligungsverfahren.

Im Zuge der Umstrukturierung der Eingliederungshilfe ist es dringend angeraten, eigene Strategien $\mathrm{zu}$ entwickeln. Modellprojekte zur Ambulantisierung und Dezentralisierung wie beispielsweise in Hamburg oder Nordrhein-Westfalen werden bundesweit beachtet und ausgewertet. Zukünftig werden sich alle Kostenträger an den Ergebnissen und Potentialen dieser Projekte orientieren. Wer sich jetzt mit einem modernen diversifizierten Dienstleistungsangebot aufstellt, sichert seine Chancen am Markt. Soviel ist sicher: Dezentralisierung, also der Aufbau von gemeindeintegrierten, flexiblen Unterstützungsangeboten für Menschen mit Behinderung, ist langfristig ohne Alternative.

Für Unternehmen der Behindertenhilfe stehen dazu nach wie vor umfängliche Fördermittel zur Verfügung. Die Identifikation der geeigneten Förderund Spendenmittel, sowie deren Beantragung gestaltet sich aufwendig und ist dennoch eine lohnende Investition. Selbstverständlich werden nur Projekte gefördert, bei denen staatliche Mittel zur Projektumsetzung nicht infrage kommen oder nachgewiesenermaßen nicht ausreichen. In der Regel kommen nur gemeinnützige, nicht kommunale Träger für eine Förderung infrage. Regelleistungen der Einrichtungen werden meist nicht zusätzlich gefördert. Weitere Merkmale der Dezentralisierung sind die Zukunftsfähigkeit sowie die Nachhaltigkeit und die klare Zielgruppenorientierung.

Aktion Mensch hat in diesem Jahr das Programm »Inklusion « aufgelegt: Intention ist die konkrete Umsetzung von Vorhaben mit inklusionsförderndem Tenor, beispielsweise durch den Aufbau von Kooperationen, Netzwerken und besonders durch die Einbindung von Menschen mit Behinderungen. Dabei werden in einem ersten Schritt zunächst nur Planungsaktivitäten und Beratung gefördert, die möglichst viele Kriterien von sozialräumlichem Arbeiten berücksichtigen. Bei »Inklusion « geht es also nicht darum, neue Einrichtungen zu schaffen, sondern Lebensqualität im Quartier und im Sozialraum als Ganzes zu denken und zu planen.

Viele Einrichtungs- und Wohngruppenleiter stellen jedoch nach einiger Zeit fest, dass die dezentrale Einrichtung im Stadtteil oder in der Gemeinde nicht automatisch zu mehr Kontakten für Menschen mit Behinderungen führt. Nachbarschaft und Netzwerke 
müssen langfristig aufgebaut und gestaltet werden. 15.000 Euro Beratungsund Planungskosten können Unternehmen der Sozialwirtschaft 2011 und 2012 beantragen, um Inklusionsprojekte zu entwickeln. Maximal 250.000 Euro können im zweiten Schritt des Programms »Inklusion « anschließend investiert werden. Dabei wird in jeder Region nur ein Projekt dieser Art gefördert.

»Rückenwind «, ein Programm des Europäischen Sozialfonds, ist trotz des relativ aufwendigen Antragsverfahrens hochinteressant im Hinblick auf die Personalentwicklungsthemen. Dezentralisierungsprojekte geraten vor allem ins Wanken, weil viele Führungskräfte und Mitarbeitende die Veränderungen im Unternehmen nicht gestalten wollen oder können. Stattdessen entsteht nicht selten ein Klima des Widerstands: Die Folge ist Innovationsstau und Stillstand. Hinzu kommen äußere Einflussfaktoren wie der Fachkräftemangel und der demographische Wandel. Mithilfe von »Rückenwind «-Projekten haben Sozialunternehmen die Möglichkeit zukunftsorientierte Maßnahmen, wie zum Thema Unternehmenskultur, selbst zu entwickeln.

Eine Grundvoraussetzung für die Fördermittelakquise ist, dass betriebswirtschaftliche und konzeptionelle Planungsschritte nachvollziehbar, beispielsweise durch einen Businessplan dargestellt werden können (vgl. Abbildung). Dieser enthält eine Analyse der aktuellen und künftigen Marktbedingungen in der Eingliederungshilfe sowie die Bedarfsermittlung für die Region. Zudem trifft der Businessplan Aussagen zur Vision und entwickelt auf dieser Basis strategische Konzepte und Angebote für aktuelle und künftige Kunden. Mithilfe einer umfänglichen Projektstruktur werden detaillierte Planungsansätze sowie Perspektiven zur Personalentwicklung und Gestaltung der internen Ressourcen entwickelt. Gute Nachnutzungskonzepte für nicht mehr genutzte Gebäude sind nicht nur aus betriebswirtschaftlicher Sicht notwendig, sondern häufig auch sinnstiftenden Charakter für das gesamte Unternehmen.

Im Rahmen der Entwicklung von Nachnutzungskonzepten lohnt es sich, mit lokalen Geldgebern ins Gespräch zu kommen und gegebenenfalls Koope-

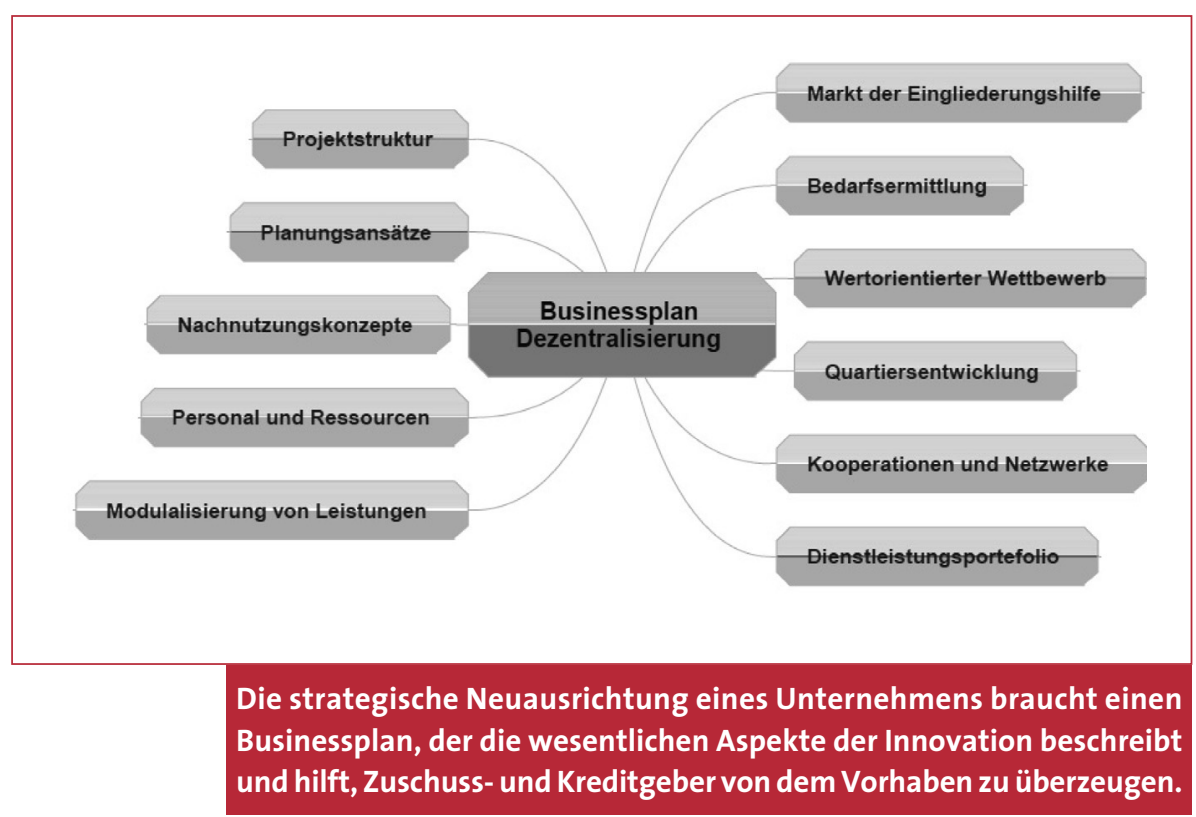

rationen einzugehen, um beispielsweise KfW-Mittel oder Mittel des Deutschen Hilfswerkes in Anspruch zu nehmen. Eigenmittel müssen in fast allen Fällen aufgebracht werden, in der Regel zwischen 10 und 50 Prozent der Gesamtsumme. Einige Fördermittelgeber finanzieren darüber hinaus Beratungsund Projektentwicklungskosten. Allein aus diesem Grund lohnt es sich für Sozialunternehmen, in das Thema Fördermittel zu investieren.

Der Aufwand ist - insbesondere bei Erstanträgen - nicht zu unterschätzen. Abhängig von der Projektgröße und dem Fördermittelgeber beträgt die Vorlaufzeit bis zu einem Jahr. Die Auswahl, Akquise und Bearbeitung von Fördermitteln sollte dementsprechend in die strategische Planung des Unternehmens eingebunden werden.

Ein Erfolgsfaktor ist die zielgerichtete Auswahl der infrage kommenden Fördermittel. Bei nationaler und internationaler Ausrichtung sind die Rahmenbedingungen häufig eng gesetzt und müssen präzise eingehalten werden. Diese Fördermittel orientieren sich an gesellschaftlichen und sozialen Trends, dementsprechend erwarten die Fördermittelgeber innovative, zukunftsfähige und lösungsorientierte Maßnahmeplanungen.

Der Aufbau von dezentralen Angeboten in der Behindertenhilfe ist per se eine große Herausforderung, die sich über mehrere Jahre hinziehen kann und einen langen Atem von allen Beteiligten fordert. Vor allem Führungskräfte kommen im Laufe der Zeit an ihre
Grenzen. Fördermittelakquise ist eine arbeitsintensive Aufgabe. Sie sollte auf Führungsebene angesiedelt sein und kontinuierlich betrieben werden, gegebenenfalls in Zusammenarbeit mit professionellen Fundraisern. Die Investition in externes oder internes Personal zahlt sich langfristig aus: So können beispielsweise gute Personalkonzepte, werte barrierefreie Wohnquartiere mithilfe von Fördermittel-Projekten gestaltet werden.

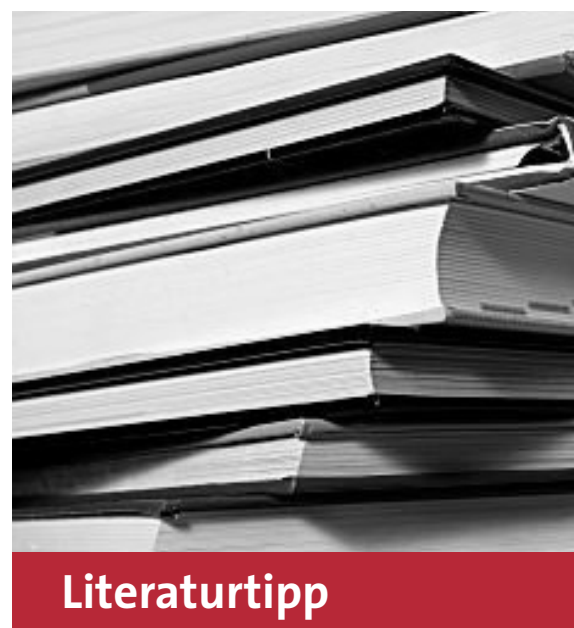

conKret Dezentralisierung: Lösungen mithilfe von Fördergeldern. Kompaktes Spezialwissen aus der Praxis für Entscheider und Führungskräfte, die sich auf strategischer Ebene mit der Konversion Ihrer Groß- und Komplexeinrichtung beschäftigen wollen. 49,- Euro. Bestellung: E-Mail info@contec.de, Leseprobe: Internet http://www.contec.de. strategische Kooperationen und lebens- 